\section{Refocusing redox biochemistry}

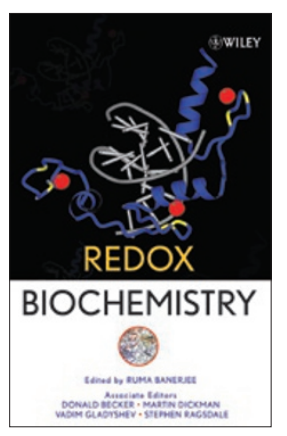

\section{Redox Biochemistry}

Edited by Ruma Banerjee and associate editors Donald Becker, Martin Dickman, Vadim Gladyshev \& Stephen Ragsdale

John Wiley \& Sons, 2007

317 pp., hardcover, $\$ 99.95$

ISBN 9780471786245

\section{Reviewed by Matthew J Wood}

How does one write a book entitled Redox Biochemistry and keep it at 300 pages? The difficulty stems from the fact that redox, an abbreviation for oxidation and reduction, is a term that commonly describes any biochemical reaction that involves the loss or gain of electrons. Redox reactions are central to all metabolic processes and thus impossible to comprehensively cover in a specialized graduate level textbook. The solution, devised by Ruma Banerjee and the co-editors of this book, is to focus on an emerging area of biology that involves redox reactions and regulation, while maintaining the emphasis on mechanistic biochemistry. Redox Biochemistry is an outgrowth of the Redox Biology Center located at the University of Nebraska at Lincoln and a graduate course collectively taught by the members of the center. As such, the text is targeted as an introductory reference to graduate students, but with its detailed and succinctly organized content, it should be of interest to any scientist needing an introduction to mechanistic redox biology.

I know from personal experience that teaching and organizing a graduate level course on redox biochemistry can present challenges. Namely, it was difficult to find a text that covered the topics needed to get a beginning graduate student up to speed. I found that using selected primary literature and review articles lacked cohesiveness. One of the clear strengths of this book is that it presents an up-to-date collection of a variety of active redox biochemistry research areas, including antioxidant enzymology, signal transduction and gene regulation. This provides a foundation for the instructor that can easily be augmented by examples from the primary literature, particularly because the book delves into areas of biochemistry that are not typically covered in more traditional biochemistry texts.

As highlighted above, the book maintains a topical theme to keep from becoming unwieldy; the focus in this case is the role of redox in the protection of cells and organisms from the collective damage of reaction oxygen species. Oxidative stress can be defined as a problem relating to cellular redox imbalance. Oxidants, in the form of reactive oxygen species (ROS) such as superoxide or hydrogen peroxide, overwhelm the normal antioxidant buffering capacity of the cell. Early work in the field elucidated

Matthew J. Wood is in the Department of Environmental Toxicology, University of California at Davis, One Shields Avenue, Davis, California 95616, USA.

e-mail:mjwood@ucdavis.edu the roles and mechanisms of antioxidant molecules in the protection against ROS. Recent research has focused on the role of ROS in regulating physiological processes and the pathology of disease processes. Organizationally, the chapters and subsections of Redox Biochemistry follow these two research themes. There are two chapters that cover the metabolic and protective roles of antioxidant molecules, cofactors and enzymes. These include individual subsections on a diversity of small molecules including glutathione, ascorbate, $\alpha$-tocopherol, lipoic acid, resveratrol and ubiquinone as well as antioxidant enzymes such as superoxide dismutase, methionine sulfoxide reductase, peroxiredoxin and catalase. Each subsection is written by an expert in the field and places emphasis on what is known about the fundamental chemical properties, redox mechanisms and metabolic role of each antioxidant molecule, cofactor or enzyme. It includes many current examples taken from primary literature. For example, Redox Biochemistry provides a mechanistic description of a recently discovered enzyme, sulfiredoxin, which functions to reduce cysteine residues that have been overoxidized to sulfinic acid.

There are also two chapters covering the expanding role of redox in regulating cellular and disease processes. The many highlights include descriptions of the role of ROS in mediating host-pathogen interactions as well as apoptotic pathways and carcinogenesis. The subsection content is not strictly limited to redox mechanisms of ROS. They also provide a redox biochemist's perspective on nitric oxide signaling and enzymology, metal homeostasis and the circadian clock.

One of the emerging areas in the field of redox biochemistry is the development of new methodologies for investigating redox enzymes and redox processes both in vitro and in live cells and organisms. Some of these discoveries are covered in the final chapter of Redox Biochemistry and highlight both experimental and computational bioinformatics approaches for investigating redox mechanisms and chemistry. Methodology development is an area that will continue to attract intense attention and focus from chemical biologists. Specific examples of redox methodology illustrated in this chapter include the use of specific chemical probes in proteomics to access the redox state of cysteine residues in vivo and the use of chemical probes to detect and quantify reactive oxygen and nitrogen species. The further development and application of chemical probes that can be used to specifically identify oxidative modification on proteins, visualize the cellular locations of ROS bursts and quantify redox potential in vivo promises to deepen the understanding of redox biochemistry in physiology and disease.

Ruma Banerjee notes that in order to keep the costs down and get this work published in a timely manner, the editors did have to make some sacrifices. For example, the book lacks primary literature references and instead provides selected references at the end of each subsection. These are intended to serve as a gateway for further reading on each topic. One sacrifice the editors did not make is in the presentation of figures and mechanisms. They achieved uniformity in all reaction mechanisms, chemical structures, protein structures and signal transduction pathways. The reasonable cost will make it an obvious choice for any graduate level course serving students in chemical or biomedical graduate programs. Additionally, new investigators would be well served to use this text as a gateway into the burgeoning and expanding area of redox biochemistry. 